\title{
Coherence in mediation activities at B1 and B2 levels
}

\section{[Quelle cohérence pour l'activité de médiation aux niveaux B1 et B2]}

\author{
Radoslaw Kucharczyk - Jaroslaw Krajka
}

DOI: $10.18355 / X L .2021 .14 .04 .06$

\begin{abstract}
The aim of this article is to analyze the issue of textual consistency in the context of mediation activities at B1 and B2 levels (in the context of French as Foreign Language).

First, we look at the question of text coherence that we analyze from both linguistic and didactic perspectives because the latter draws a lot from the contributions of pragmatics. As our research is in the context of teaching/learning foreign languages, we treat cohesion as one of the linguistic phenomena impacting coherence. In the following, our text deals with mediation as one of the activities to be developed in the language class. We are convinced that this type of language activity requires the learner to master logical connectors, which could make the mediated text coherent and - consequently - faithful to the source text. It is in this perspective that our research is situated, which concludes the article. We analyzed students' certification exams in French (at B1 and B2 levels) to see which logical connectors they used to mediate an oral and written. To do this, we developed a corpus that was submitted for analysis. The results of our study show that the list of logical connectors to which the students used is far from being representative for the given levels (B1 and B2 in this case), which allowed us to formulate pedagogical guidelines and questions for future research.
\end{abstract}

Key words: coherence, logical connectors, mediation, certification exam, FLE

\section{Résumé}

L'objectif de cet article est d'analyser la question de la cohérence textuelle dans le cadre des activités de médiation aux niveaux B1 et B2 (dans le cadre du français langue étrangère). Tout d'abord, nous nous penchons sur la question de la cohérence des textes que nous analysons à la fois dans une perspective linguistique et didactique, car cette dernière puise beaucoup dans les apports de la pragmatique. Notre recherche s'inscrivant dans le cadre de l'enseignement/apprentissage des langues étrangères, nous traitons la cohésion comme l'un des phénomènes linguistiques impactant la cohérence. Dans ce qui suit, notre texte traite de la médiation comme l'une des activités à développer en classe de langue. Nous sommes convaincus que ce type d'activité langagière nécessite que l'apprenant maîtrise des connecteurs logiques qui puissent rendre le texte médiatisé cohérent et - par conséquent - fidèle au texte source. C'est dans cette perspective que se situe notre recherche qui conclut l'article. Nous avons analysé les examens de certification des étudiants en français (aux niveaux B1 et B2) pour voir quels connecteurs logiques ils utilisaient pour médier un oral et un écrit. Pour ce faire, nous avons élaboré un corpus qui a été soumis à l'analyse. Les résultats de notre étude montrent que la liste des connecteurs logiques auxquels les étudiants ont utilisé est loin d'être représentative pour les niveaux donnés (B1 et B2 dans ce cas), ce qui nous a permis de formuler des orientations pédagogiques et des questions pour de futures recherches.

Mots clés : cohérence, connecteurs logiques, médiation, examen de certification, FLE

XLinguae, Volume 14 Issue 4, October 2021, ISSN 1337-8384, eISSN 2453-711X 


\section{Introduction}

L'objectif du présent article est d'analyser la question de la cohérence textuelle dans le cadre des activités de médiation au niveau B1 et B2 (contexte du FLE).

D'abord, nous nous penchons sur la question de la cohérence des textes que nous analysons dans une perspective aussi bien linguistique que didactique, car cette dernière puise beaucoup dans les apports de la pragmatique. Comme notre recherche se situe dans le cadre de l'enseignement/apprentissage des langues étrangères, nous traitons la cohésion en tant qu'un des phénomènes linguistiques impactant la cohérence. Dans la suite, notre texte traite de la médiation en tant qu'une des activités qui sont à développer en classe de langue. Nous sommes persuadés que ce type d'activités langagières exige de la part de l'apprenant la maîtrise de connecteurs logiques qui pourraient rendre le texte médié cohérent et - par voie de conséquence fidèle au texte source. C'est dans cette optique que se situe notre recherche qui clôt l'article. Nous avons analysé des copies d'étudiants qui se sont présentés à l'examen certificatif en langue française (niveau B1 et B2) pour examiner à l'aide de quels connecteurs logiques ils médient un texte oral et écrit, ce qui constituait le noyau dur de la tâche de l'examen. Pour ce faire, nous avons élaboré un corpus qui a été soumis à analyse. Les résultats de notre étude montrent que la liste de connecteurs logiques auxquels les étudiants ont recouru est loin d'être représentative pour les niveaux donnés (B1 et B2 en l'occurrence), ce qui nous a permis de formuler des pistes pour des démarches didactiques appropriées et des questions pour des recherches à l'avenir.

\section{Qu'est-ce qu'on entend par la cohérence du texte? \\ 1.1 L'approche linguistique}

La notion de cohérence textuelle n'est pas une notion nouvelle, ni pour la linguistique ni pour la didactique des langues étrangères. En effet, elle puise ses racines dans la pragmatique, ou, plus précisément, dans la linguistique des textes et l'analyse du discours (Cuq, 2003 : 46). La notion de cohérence renvoie à la structuration du texte qui peut être due soit à son fonctionnement interne qui est conditionné par les structures langagières au sens large du terme (structures grammaticales et lexicales) soit par les marqueurs discursifs engendrés par le type de texte donné. En résumé, étudier la cohérence textuelle revient à étudier comment est organisée la progression thématique du texte et à l'aide de quels marqueurs discursifs ladite progression est exprimée. Pour rapprocher mieux la question de cohérence, il nous semble important de nous référer aux quatre règles de cohérence de Charolles (cité par Salles, $2006: 2$ ) qui se présentent comme suit :

- méta-règle de répétition qui accentue la nécessité de la progression linéaire du texte soit au niveau macro (ensemble du texte) soit au niveau micro (cohérence interne) ;

- méta-règle de progression qui met en relief la nécessité d'un renouvellement sémantiques des éléments textuels pour faire progresser le texte ;

- méta-règle de non-contradiction qui impose que le déroulement du texte n'impose pas de contradictions sémantiques par rapport au contenu antérieur ou postérieur ;

- méta-règle de relation qui exige que les éléments dénotant le monde soient liés directement (sont distinguées alors des relations de cohérence telles que la cause-conséquence, l'élaboration, le contraste, l'exemplification, la généralisation, etc.).

Du point de vue de nos réflexions, il nous semble que c'est la règle de répétition et celle de relation qui devraient attirer notre attention. Ainsi, arrivons-nous à la notion de cohésion du texte dont la fonction est de mettre en exergue les relations entre 
différentes parties du texte au niveau local (Alkhabit, 2012 : 54). D'où l'importance accordée aux mots dits de liaisons qui structurent le texte. En effet, il n'est possible ni de marquer la progression du texte ni de mettre en évidence les relations entre différents éléments (ou passages) du texte sans recourir à des articulateurs du discours ou, en d'autres termes, des connecteurs logiques.

Comme le remarque M. Alkhatib (2012:54), « les connecteurs sont des phrases, des groupes de mots ou des mots qui indiquent l'organisation d'un texte. Ils annoncent un nouveau passage, résument, marquent une transition, concluent ... Ils sont souvent placés au début ou à la fin d'un paragraphe. Ils peuvent indiquer que, dans une même séquence textuelle, on change de lieu, de temps, d'aspect traité, d'argument, etc. ». Nous pouvons donc admettre que la notion de connecteurs logiques est une notion vaste englobant des phénomènes linguistiques différents qui se placent entre les moyens syntaxiques au sens large du terme et les moyens logico-syntaxiques (Attabi, 2012 : 84). En d'autres termes, les connecteurs logiques peuvent structurer le texte soit au niveau minimal pour signaler les relations entre deux propositions soit au niveau global pour signaler les relations entre différentes parties du même texte. Il s'ensuit alors que la fonction majeure des connecteurs logiques est celle d'organiser le texte et de rendre possible son interprétation. On parle alors de deux fonctions de connecteurs logiques : celle de balisage (structurer les phrases ou différentes parties du texte) et celle d'ancrage (guider l'interprétation du texte). Dans la suite du texte, nous traitons de la cohésion, qui se réalise avant tout par le biais du recours aux connecteurs logiques, en tant qu'une des composantes de la cohérence.

\subsection{L'approche didactique}

En didactique des langues étrangères, la question de cohérence textuelle apparaît avec l'approche communicative dans les années 70/80 du XX ${ }^{\text {ème }}$ siècle où les recherches menées en classe de langue s'inspirent des apports de la pragmatique. En effet, même si c'est la compétence de communication qui se place au cœur des réflexions didactiques portant sur l'approche communicative, elle est décortiquée en différentes sous-compétences qui doivent trouver leur place légitime en classe de langue. En analysant différents modèles de ladite compétence qui tentent de la décrire de la façon la plus détaillée possible (Zając, 2004: 18-24), nous pouvons constater que la composante relative à l'organisation des textes y est toujours présente. Peu importe si elle se cache dernière le vocable «la compétence discursive », «la compétence d'organisation » ou encore « la compétence sémiolinguistique », elle renvoie toujours à l'organisation, aussi bien interne qu'externe, des textes qui sont toujours contextualisés par différents paramètres de la situation de communication dans laquelle ils apparaissent. Ainsi, travailler sur différents types de discours, en les analysant par rapport à leur organisation et puis en les reproduisant tant à l'oral qu'à l'écrit, devient l'un des objectifs du processus d'enseignement/apprentissage des langues qui visent la communication. Comme le remarquent à juste titre J.-P. Cuq et I. Gruca (2012 : 266) «savoir communiquer ne se réduit en aucun cas à la simple connaissance de la langue, mais implique d'une manière ou d'autre la connaissance des règles d'emploi de cette langue mises en valeur par les [...] composantes qui forment la compétence de communication ». La notion de cohérence gagne encore en importance avec la parution du Cadre européen commun de référence (désormais le CECRL) (2001) dont les concepteurs, tout en accentuant l'ancrage social de l'utilisation des langues, distinguent la compétence pragmatique en tant qu'une des compétences faisant partie de la compétence à communiquer langagièrement. Elle est définie de la manière suivante : "La compétence pragmatique recouvre l'utilisation fonctionnelle des ressources de la langue [...] en s'appuyant sur des scénarios ou des scripts d'échanges interactionnels. Elle renvoie également à la maîtrise du discours, à sa cohésion et à sa cohérence, au repérage des types de genres textuels, des effets

XLinguae, Volume 14 Issue 4, October 2021, ISSN 1337-8384, eISSN 2453-711X 
d'ironie, de parodie. » (CECRL, $2001: 18)$. Il découle du passage qui précède que la cohérence (y compris aussi la cohésion qui, comme nous l'avons constaté plus haut, la conditionne) est un élément crucial de la compétence pragmatique. En effet, elle est strictement liée à la compétence discursive ${ }^{1}$ qui permet à l'apprenant de structurer son discours qu'il soit oral ou écrit. Elle englobe des savoirs et savoir-faire tels que la connaissance de l'organisation des phrases et leurs composantes, la capacité à maîtriser les phrases (thème/rhème; information donnée/information nouvelle ; enchaînement des phrases suite à différentes relations logiques), la capacité à gérer et structurer le discours (organisation thématique, cohérence et cohésion, organisation logique, style et registre, efficacité rhétorique, principe coopératif). La même idée est reprise dans le volume complémentaire du CECRL (désormais le CECRL VC) de 2018 dont les auteurs accordent encore plus d'attention à la compétence pragmatique et discursive de même qu'à la cohérence et la cohésion. En effet, si « la cohésion et la cohérence fonctionnent à la fois au niveau de la phrase /de l'énoncé et au niveau du texte entier»(CECRL VC, 2018: 149), les démarches didactiques entreprises en classe doivent cibler les savoir-faire suivants :

- relier les mots et les éléments à l'aide de connecteurs logiques et temporels ;

- $\quad$ structurer le texte à l'aide des paragraphes ;

- recourir à différents outils linguistiques pour choisir des connecteurs précis. Il n'est pas donc étonnant de voir qu'il existe une échelle de descripteurs qui tentent de décrire la cohérence et la cohésion. Nous la présentons ci-dessous :

\section{Tableau 1 La cohérence selon le CECR}

\begin{tabular}{|c|l|}
\hline C2 & $\begin{array}{l}\text { Peut créer un texte cohérent et cohésif en utilisant de manière complète et appropriée les structures } \\
\text { organisationnelles adéquates et une grande variété d'articulateurs. }\end{array}$ \\
\hline C1 & $\begin{array}{l}\text { Peut produire un texte clair, fluide et bien structuré, démontrant un usage contrôlé de moyens linguistiques de } \\
\text { structuration et d'articulation. } \\
\text { Peut produire un texte bien organisé et cohérent en utilisant une variété d'articulateurs et de schémas } \\
\text { d'organisation. }\end{array}$ \\
\hline B2 & $\begin{array}{l}\text { Peut utiliser avec efficacité une grande variété de mots de liaison pour marquer clairement les relations entre } \\
\text { les idées. } \\
\text { Peut utiliser un nombre limité d'articulateurs pour relier ses énoncés dans un discours clair et cohérent, bien } \\
\text { qu'il puisse y avoir quelques « sauts » dans une longue intervention. } \\
\text { Peut produire un texte en général bien organisé et cohérent, utilisant toute une gamme de mots de liaison et } \\
\text { d'articulateurs. } \\
\text { Peut organiser des textes longs en paragraphes logiques. }\end{array}$ \\
\hline B1 & $\begin{array}{l}\text { Peut introduire un contre argument dans un texte simple discursif (par exemple avec " cependant »). } \\
\text { Peut relier une série d'éléments courts, simples et distincts en une suite linéaire de points qui s'enchainent. } \\
\text { Peut élaborer, dans un récit, des phrases assez longues et les relier entre elles en utilisant un nombre limité } \\
\text { d'articulateurs. } \\
\text { Peut créer des sauts de paragraphes simples et logiques dans un texte assez long. }\end{array}$ \\
\hline A2 & $\begin{array}{l}\text { Peut utiliser les articulations les plus fréquentes pour relier des énoncés afin de raconter une histoire ou décrire } \\
\text { quelque chose sous forme d'une simple liste de points. } \\
\text { Peut relier des groupes de mots avec des connecteurs simples tels que « et », « mais » et " parce que ». }\end{array}$ \\
\hline A1 & Peut relier des groupes de mots avec des connecteurs élémentaires tels que « et » ou « mais ». \\
\hline Pré-A1 & Pas de descripteur disponible \\
\hline
\end{tabular}

Il découle de ce qui précède que la cohérence et la cohésion sont des éléments qui devraient être travaillés consciemment lors du processus d'enseignement/apprentissage des langues étrangères. D'un côté, la tâche de l'enseignant est d'apprendre aux élèves comment structurer des phrases et puis - à une étape plus avancée - le texte/le discours, et de l'autre, d'amener les apprenants à la précision de leurs textes suite à l'utilisation de connecteurs logiques de plus en plus nuancés.

\footnotetext{
${ }^{1}$ Rappelons que la compétence discursive est, selon les concepteurs du CECRL, une des souscompétences - à côté de la compétence fonctionnelle et de la compétence de conception schématique - qui forme la compétence pragmatique.
} 


\section{La cohérence du texte dans les activités de médiation}

La parution du CECRL (2001) a non seulement rendu le processus d'enseignement/apprentissage et évaluation des langues plus homogène, mais elle a aussi mis au premier plan la médiation en tant qu'une des activités langagières qui permettent à l'apprenant de développer ses compétences, aussi bien générales que langagières. Les auteurs du CECRL constatent que «dans les activités de médiation, l'utilisateur de la langue n'a pas à exprimer sa pensée mais doit tout simplement jouer le rôle d'intermédiaire entre des interlocuteurs incapables de se comprendre en direct » (CECRL, 2001: 71). La définition citée ci-dessus - qui est loin d'être exhaustive - réduit les activités de médiation à une simple retransmission des paroles d'un tiers ce qui revalorise les activités de traduction/interprétation en classe de langue (Rober et Rosen, 2010 : 145). En effet, parmi les activités de médiation qui sont distinguées dans le $C E C R L$, se trouvent les activités suivantes : l'interprétation, la traduction, le résumé et la reformulation qui - ancrées dans le contexte social de l'utilisation de la langue - sortent de l'oubli didactique revêtues d'une autre forme. Force est quand-même de constater que ladite activité reste marginalisée par rapport aux autres activités décrites minutieusement dans le référentiel. Cependant, ce n'est qu'en 2018, suite à la parution du CECRL VC que la médiation devient une activité langagière traitée avec beaucoup de précision qui retrouve sa place légitime en classe de langue. En effet, on part du principe que la médiation ne s'appuie pas seulement sur les compétences langagières de l'apprenant (qui sont nécessaires pour les activités de traduction/interprétation et reformulation) mais aussi sur les compétences générales. Ainsi, peuvent être distinguées la médiation rationnelle et la médiation cognitive (Goullier, 2019 : 101). La première recouvre la capacité à gérer des relations interpersonnelles, ce qui sous-entend l'établissement d'une ambiance positive pour faciliter les échanges et la coopération. Quant à la deuxième, elle vise la capacité à faciliter l'accès au sens (sens du texte ou sens du comportement) au cas où un tiers n'est pas compétent soit en langue soit dans un sujet abordé lors de la communication. Il est donc question de deux modes de médiation possibles: (re)construire ou retransmettre le sens d'un texte. Ainsi, «le cœur de la médiation, objet d'apprentissage, consiste à la fois à identifier les obstacles d'ordre culturel à la compréhension d'un énoncé, d'un texte ou d'un comportement et à respecter la dimension linguistique du message ou du texte éventuel concerné. » (Goullier, 2019 : 102). Ce point de vue est repris dans le CECRL VC où - comme nous l'avons déjà constaté - les activités de médiation sont valorisées. Préparer l'apprenant à devenir médiateur revient à lui fournir des moyens pour qu'il soit capable de construire des passerelles afin de retransmettre du sens soit dans la même soit dans une autre langue dans des contextes variés : social, pédagogique, linguistique ou professionnel (CECRL $V C, 2019$ : 106). Il en résulte que la langue devient un outil qui rend possible les processus suivants :

- créer l'espace et les conditions pour apprendre/communiquer ;

- coopérer pour construire un nouveau sens ;

- $\quad$ encourager un tiers à construire et à comprendre un nouveau sens ;

- transmettre des informations nouvelles de façon appropriée.

Ainsi, sont distingués trois groupes de médiation : la médiation de textes, la médiation de concepts et la médiation de communication. Le premier groupe vise directement le traitement des textes et - par voie de conséquence - englobe des activités telles que, par exemple, transmettre des informations spécifiques (à l'oral et à l'écrit), traduire/interpréter un texte, prendre des notes ou exprimer une réaction personnelle à l'égard d'un texte. Quant à la médiation de concepts, elle est focalisée sur la capacité à travailler en équipe pour faciliter l'accès au savoir et aux concepts. D'où l'accent mis sur les habiletés suivantes : savoir travailler de façon coopérative ou mener un 
travail de groupe en construisant, entre autres, du sens. Last but not least, la médiation de communication a pour objectif de «faciliter la compréhension et de façonner une communication réussie entre les utilisateurs/apprenants qui ont des perspectives individuelles, socioculturelles, sociolinguistiques ou intellectuelles éventuellement différentes. » (CECRL VC, 2018 : 110). Pour ces raisons, l'enseignant de langue est censé développer auprès des apprenant des compétences telles que savoir établir un espace pluriculturel ou faciliter la communication dans des situations délicates et des désaccords. Il est aussi important de signaler que, pour chaque type de médiation, les concepteurs du CECRL VC proposent des échelles de compétences pour les activités de médiation qui « ne sont pas des outils destinés exclusivement à l'évaluation mais peuvent se révéler très utiles aussi pour penser la progression dans les entraînements à cette activité (...)»(Goullier, 2019: 105). À part les descripteurs des trois groupes d'activités de médiation, le référentiel propose également une typologie de stratégies qui soutiennent le développement de ladite activité (CECR VC, 2018 : 132-133) : stratégies pour expliquer un nouveau concept (relier à un savoir préalable, adapter son langage, décomposer une information compliquée) ou stratégies pour simplifier un texte (amplifier un texte dense, élaguer un texte). Bref, la médiation devient une activité langagière à part entière dont les différentes facettes devraient se retrouver en classe de langue. La question que nous nous posons alors est celle de savoir comment marier la compétence discursive qui englobe - nous l'avons déjà remarqué - les savoirs et les savoir-faire relatifs à la structuration du texte et du discours et les activités de médiation, surtout celle de texte. Nous tenterons de répondre à cette question dans la suite du présent article où nous présenterons l'analyse de deux corpora d'apprenants (niveau B1 et B2) qui ont été confrontés à des activités de médiation lors de leur examen certificatif de langue française à l'Université de Varsovie en Pologne.

\section{L'étude empirique}

\subsection{Les corpus en didactique des langues étrangères}

Les recherches basées sur les corpus sont relativement nombreuses en didactique des langues étrangères : d'un côté, il existe des corpus peu denses adaptés aux besoins des élèves (Aston, 1997), de l'autre, des corpus représentatifs basés sur le phénomène de concordance (Bernardini, 2000; de Schryver, 2002), utilisés dans l'amélioration de la compétence écrite aux niveaux inférieurs (Gaskell \& Cobb, 2004; Yoon \& Hirvela, 2004) et avancés (Chambers \& O'Sullivan, 2004) ou des corpus présentant certaines règles d'usage de la grammaire (Hadley, 2002) ou encore des inférences de ces règles (St. John, 2001). Il va aussi de soi qu'utiliser les corpus en didactique des langues étrangères ouvre la voie pour les études sur l'apprentissage du lexique (Cobb, 1997, 1998) et les études portant sur la compréhension des écrits.

Force est quand-même de constater que l'analyse des corpus doit être toujours contextualisée par le recours aux dictionnaires, à des textes troués, aux hypertextes et à des bases de données incluant des activités autocorrectives (Cobb et al., 2001; Horst et al., 2005; voir aussi Lextutor's routines - http://www.lextutor.ca). Nombreuses sont aussi les recherches examinant la relation entre l'efficacité de la consultation des corpus et l'enseignement/apprentissage stratégique des langues (Chambers, 2005; Kennedy, Miceli, 2001; St. John, 2001) qui montrent la nécessité d'introduire consciemment et progressivement ces outils en classe de langue. Récemment, apparaissent des recherches (Lee et al. 2017) qui examinent la compréhension des textes via des corpus glosés sur la concordance.

D'autres études (par exemple, Marinov, 2013) montrent que les tâches d'apprentissage basées sur le corpus développent la conscience linguistique et impactent positivement la formation des futurs enseignants de langues. 
Du point de vue du présent article, il nous semble important d'accentuer le rôle de corpus d'apprenants construits par les enseignants eux-mêmes (Lee, Swales, 2006) dans le processus d'enseignement/apprentissage des langues. En effet, les corpus en question semblent être utiles pour examiner le niveau de langue actuel chez les apprenants afin de choisir les activités et les techniques de classe appropriées. En ce sens, le corpus en question peut être un outil complémentaire pour évaluer les tests de compétence linguistique : il nous permet d'examiner si le candidat recourt à des structures censées apparaître au niveau donné.

\subsection{L'objectif de l'étude}

L'objectif de notre recherche était d'examiner avec quelle intensité les articulateurs logiques exprimant le but, la cause, la conséquence, la concession et l'opposition, censés apparaître aux niveaux $\mathrm{B} 1$ et $\mathrm{B} 2$ selon le $C E C R L$, sont présents dans les copies des étudiants qui passent leur examen de certification (obligatoire à la fin des études de $1^{\text {er }}$ cycle) soit au niveau B1 soit au niveau B2. Pour ce faire, nous avons établi - en nous appuyant sur des manuels de grammaire visant les deux niveaux ${ }^{2}-$ une liste de connecteurs logiques que nous présentons ci-dessous :

\footnotetext{
${ }^{2}$ Akyüz, A. et al. (2019). Exercices de grammaire B1. Paris : Hachette FLE. ; Akyüz, A. et al. (2019). Exercices de grammaire B2. Paris : Hachette FLE. ; Caquineau-Gündüz, M-P. et al. (2005). Les 500 exercices de grammaire. Niveau B1. Paris : Hachette FLE. : Caquineau-Gündüz, M-P. et al. (2007). Les 500 exercices de grammaire. Niveau B2. Paris : Hachette FLE.
} 
Tableau 2: Les connecteurs logiques (B1 et B2)

\begin{tabular}{|c|c|c|}
\hline relation logique & articulateurs - nivean Bl & articulateurs - viveau B2 \\
\hline but & $\begin{array}{ll}\text { - } & \text { pour } \\
\text { - } & \text { afin de } \\
\text { - } & \text { pour que } \\
\text { - } & \text { afin que }\end{array}$ & $\begin{array}{l}\text { - de maniére que + subjonctif } \\
\text { - de façon que + subjonctif } \\
\text { - de sorte que + subjonctif } \\
\text { - de maniére à + infinitif } \\
\text { - de façon a + infinitif } \\
\text { - de sorte de + infinitif } \\
\text { - dans lintention de + } \\
\text { - infinitif } \\
\text { - dans le but de + infinitif } \\
\text { - de vue de + infinitifinom } \\
\text { - de peur que + subjonctif } \\
\text { - de crainte de + infinitif } \\
\text { - de peur de + infinitif }\end{array}$ \\
\hline cause & $\begin{array}{l}\text { - à cause de } \\
\text { - gráce à } \\
\text { - parce que } \\
\text { - car } \\
\text { - en raison de } \\
\text { - prisque } \\
\text { - comme } \\
\text { - en raison de }\end{array}$ & $\begin{array}{l}\text { - } \text { par suite de } \\
\text { - } \text { compte tenu de } \\
\text { - étant donné que } \\
\text { - } \text { va } \\
\text { - en effet } \\
\text { - faute de } \\
\text { - à force de } \\
\text { - par manque de } \\
\text { - par + nom sans article } \\
\text { - pour + nominfinitif passé } \\
\text { - suite à + nom } \\
\text { - à la suite de + nom } \\
\text { - sous prétexte de + nom } / \\
\text { infinitif }\end{array}$ \\
\hline conséquence & $\begin{array}{ll}\text { - } & \text { alors } \\
\text { - } & \text { donc } \\
\text { - } & \text { par conséquence } \\
\text { - c'est pourquoi } \\
\text { - c'est pour ça que } \\
\text { - } & \text { si bien que } \\
\end{array}$ & $\begin{array}{l}\text { - de ce fait } \\
\text { - d'où + nom } \\
\text { - ce qui explique + nom } \\
\text { - ce qui explique + indicatif } \\
\text { - du coup }\end{array}$ \\
\hline opposition & $\begin{array}{l}\text { - alors que } \\
\text { - au lieu de + infinitif } \\
\text { - mais }\end{array}$ & $\begin{array}{ll}\text { - } & \text { par contre } \\
\text { - } & \text { en revanche } \\
\text { - } & \text { au contraire } \\
\text { - } & \text { au contraire de + } \\
\text { nom/pronom } \\
\text { - } \\
\text { à l'inverse de + } \\
\text { nom/pronom } \\
\text { - à l'opposé de + } \\
\text { nom/pronom } \\
\text { - contrairement à + } \\
\text { nom/pronom }\end{array}$ \\
\hline concession & $\begin{array}{l}\text { - } \text { pourtant } \\
\text { - } \text { cependant } \\
\text { - malgré + nom } \\
\text { - } \text { bême si } \\
\text { - } \text { avoir que + subjonctif + infinitif }\end{array}$ & $\begin{array}{l}\text { - cela étant } \\
\text { - } \text { toutefois } \\
\text { - néanmoins } \\
\text { - } \text { en dépit de + nom /prénom } \\
\text { - } \text { quitte à + infinitif } \\
\text { - } \\
\text { - } \text { quans que + subjonctio à ce que + subjonctif }\end{array}$ \\
\hline
\end{tabular}

Nous partons du principe que les étudiants du niveau B2 - où une certaine précision linguistique est nécessaire - vont recourir non seulement à des connecteurs «basiques » (ceux du niveau B1) mais aussi à des connecteurs plus recherchés pour exprimer leurs pensées de manière plus ordonnée. Ainsi, la question qui a guidé notre recherche était formulée de la façon suivante: comment les étudiants passant l'examen de certification réalisent-ils des intentions communicatives données ? Recourent-ils consciemment et efficacement aux articulateurs prévus pour le niveau donné ou évitent-ils les articulateurs d'un niveau plus avancé en se limitant à des articulateurs «basiques"? Nous nous sommes également intéressés à la question de savoir à quel point les articulateurs logiques utilisés par les étudiants pour exprimer le but, la cause, la conséquence, la concession et l'opposition étaient variés. 


\subsection{Le corpus analysé}

Le corpus d'apprenants analysé dans le présent article est basé sur les copies des apprenants de l'Université de Varsovie en Pologne se présentant à l'examen certificatif de français (niveaux B1 et B2) organisé au mois de février 2021, à cause de la pandémie, sur la plateforme Moodle. La tâche à laquelle les étudiants ont été confrontés consistait à rédiger un texte en se basant sur les informations incluses dans un enregistrement et un article à lire. Ci-contre, nous présentons les consignes de l'examen ${ }^{3}$ :

Niveau B1

Écoutez le fichier ci-joint deux fois et lisez le texte joint à la tâche. Remplissez ensuite la lettre ci-dessous afin d'avoir un texte cohérent.

Dans la partie 1. Rapportez-vous aux informations contenues dans l'enregistrement. Attention! Les réponses doivent être complètes et formulées avec vos propres mots. La réécriture de phrases entières à partir de l'enregistrement ne sera pas considérée comme une bonne réponse.

Dans la partie 2. Complétez les extraits de phrase manquants en vous référant aux informations contenues dans l'article. Veuillez noter que le libellél les phrases réécrites /copiées seront considérés comme une réponse incorrecte.

Dans la partie 3. Écrivez ce qui vous pose le plus de difficultés quand vous parlez en public et comment, selon vous, on peut surmonter ces difficultés. Attention !! Votre texte dans la 3ème partie du texte devrait contenir 150-200 mots.

Poznań, le 3 décembre 2020

Chère Céline,

Je viens de recevoir ta lettre. Je sais que tu es occupée à préparer une présentation pour la conférence dont tu me parles et que cela te stresse. Ne t'inquiète pas, c'est normal. Je sais que parler en public n'est pas facile. Mais je peux t'aider.

(1) Récemment, j'ai écouté une émission de radio intéressante où un psychologue a parlé des automatismes qui nous aident à prendre la parole en public.

Tout d'abord, il faut maîtriser la respiration qui aide à gérer le stress.

Ensuite, il a parlé du sourire parce que

Le dynamisme est aussi important

Le spécialiste a bien insisté sur le fait que nous devons regarder notre public.

Finalement, selon lui, il ne faut pas trop bouger parce que

(2) Après avoir écouté cette émission, j'ai aussi trouvé un article qui présente des conseils pour prendre la parole lors de visioconférence.

\footnotetext{
${ }^{3}$ source : les supports du Conseil de certification des compétences en LE de l'Université de
} Varsovie.

XLinguae, Volume 14 Issue 4, October 2021, ISSN 1337-8384, eISSN 2453-711X 


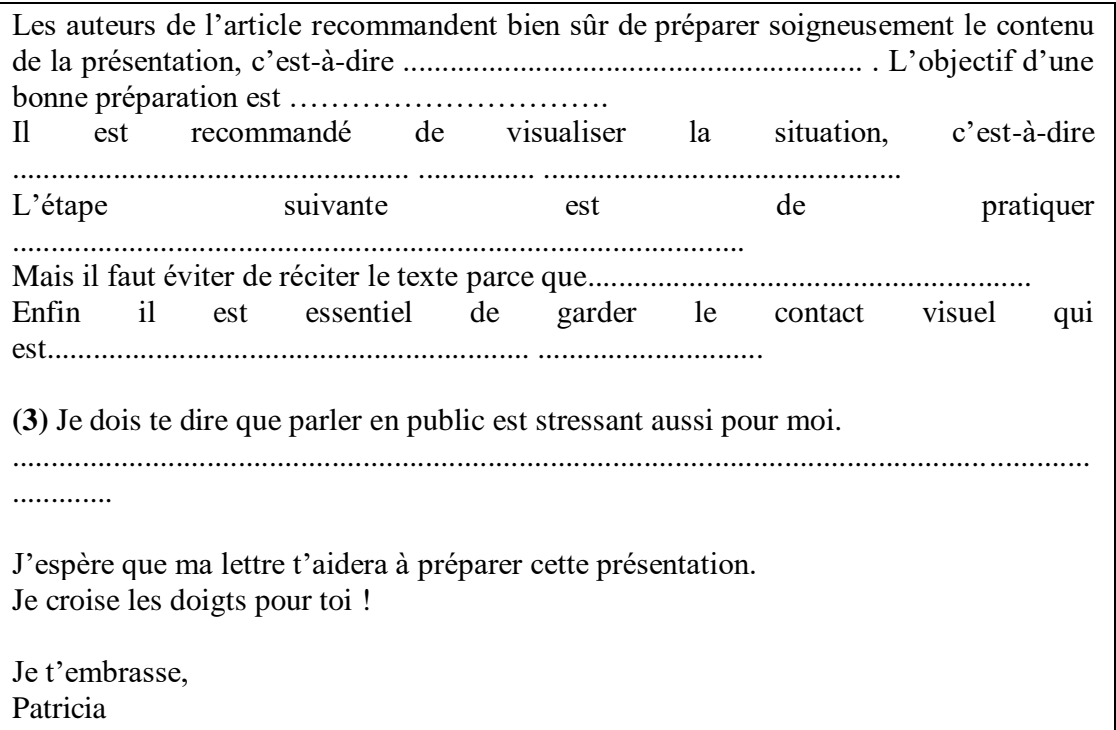

\section{Niveau B2}

La voiture électrique, véhicule le plus écologique ou fausse solution miracle? Si la production de sa batterie alourdit son bilan écologique, elle devient de plus en plus intéressante à l'usage au fil des kilomètres. On vous a demandé d'examiner les avantages et les inconvénients des véhicules électriques. Les avis sont partagés. Rédigez un bref texte dans lequel

1. vous ferez attention aux éléments importants soulevés pour et contre les véhicules électriques sur la base des textes présentés

2. vous indiquerez l'option qui vous semble la meilleure et vous justifierez votre choix.

Votre rapport doit contenir entre 250 et 300 mots. Assurez-vous de vous référer aux deux parties (enregistrement et article).

Dans la partie 1, il vous est demandé de présenter, avec vos propres mots, des arguments qui apparaissent dans les deux textes (minimum 3 de chacun). Dans la partie 2, il s'agit de présenter et de justifier votre opinion. Vous recevrez des points pour avoir démontré votre compréhension des textes (partie 1) et pour avoir exprimé votre opinion de manière claire et complète (partie 2).

Il résulte de ce qui précède que les étudiants ont dû réaliser une activité de médiation de texte qui exigeait de leur part les compétences suivantes (CECRL 2018 : 109) :

- $\quad$ transmettre des informations spécifiques ;

- $\quad$ traiter un texte ;

- $\quad$ prendre des notes ;

- $\quad$ exprimer une réaction personnelle.

Le corpus soumis à l'analyse se compose de toutes les copies des étudiants se présentant à l'examen, à savoir 21 copies au niveau B1 et 17 copies au niveau B2. Le sous-corpus B1 contient 11264 mots (1916 types, y compris 488 à fréquence supérieure à 2), alors que le sous-corpus B2 contient 5007 mots (1 299 types, y compris 306 à fréquence supérieure à 2). Malgré une faible différence dans le nombre 
de candidats $(\mathrm{B} 1=21$ personnes, $\mathrm{B} 2=17$ personnes), le sous-corpus $\mathrm{B} 1$ est deux fois plus grand que le sous-corpus B2 ce qui résulte probablement du fait que les étudiants B2 s'expriment avec plus de précision, ce qui sous-entend le recours à moins de mots pour réaliser la tâche imposée par la consigne de l'examen. De plus, la consigne pour le niveau B1 (rédiger une lettre) demandait aux apprenants de compléter une lettre dont les fragments étaient donnés, ce qui explique probablement le volume plus élevé du sous-corpus B1.

$\mathrm{Si}$ on prend en considération la forme finale des deux sous-corpus, il n'est pas étonnant de constater que les articulateurs sont surreprésentés dans le sous-corpus B1 par rapport au sous-corpus B2. Il résulte de ce qui précède que, dans nos analyses, nous éviterons de comparer les deux groupes d'étudiants se présentant à l'examen certificative. De plus, il nous semble également important de prendre en considération la nature des tâches que les étudiants ont dû effectuer lors de l'examen. Néanmoins, il sera intéressant d'examiner si les étudiants au niveau B1 essaient de recourir à des articulateurs du niveau supérieur (niveau B2 en l'occurrence) et si les étudiants au niveau B2 utilisent des articulateurs du niveau concerné (même de façon limitée) ou plutôt s'ils se limitent à des outils linguistiques de base pour réussir la tâche de l'examen.

\subsection{Les étapes de la recherche}

Dans la première étape de la création du corpus, nous avons extrait des textes des étudiants stockés sur la plateforme Moodle : aussi bien les textes au niveau B1 que ceux au niveau B2. Ensuite, nous avons compilé plusieurs versions de notre corpus d'apprenants : le corpus général (niveau B1 et niveau B2), le sous-corpus B1 et le sous-corpus B2 et les sous-corpus B1_LOW, B1_MID, B1_HIGH, B2_LOW, B2_MID i B2_HIGH qui prenaient en considération les résultats finaux de l'examen. Cependant, compte-tenu du nombre restreint de copies, de même que de la répartition inégale des scores, nous avons renoncé à analyser les articulateurs selon les niveaux visés. Nous avons alors décidé de les analyser dans les sous-corpus séparés : celui du niveau B1 et celui du niveau B2.

Dans la deuxième phase de la création du corpus, nous avons analysé les textes des étudiants pour éliminer les fragments qui ne réalisaient pas la tâche : dans le souscorpus B2 ces cas étaient absents, alors que dans le sous-corpus B1 apparaissaient des reprises de la consigne ou des phrases dans une autre langue (en anglais le plus souvent) qui constituaient une sorte d'explication aux correcteurs.

Dans la troisième phase, nous avons effectué l'analyse de la fréquence de tous les mots présents dans les deux corpus, tout en accordant une attention particulière aux articulateurs logiques que nous avons présentés dans le tableau ci-dessus en tant que mots de liaison caractéristiques pour les niveaux B1 et B2. C'est à cette étape que nous avons eu non seulement la possibilité de constater certaines régularités relatives à la distribution des articulateurs dans les deux sous-corpus mais aussi de diagnostiquer des formes mal employées ${ }^{4}$.

Au départ de la recherche, nous avons lancé une requête de concordance pour extraire des deux sous-corpus des connecteurs logiques censés apparaître aux niveaux concernés. Nous avons également éliminé des articulateurs mal employés et - en même temps - nous avons marqué des formes mal utilisées mais rendant la relation donnée communicative pour l'analyse qualitative.

Enfin, nous avons exporté les données obtenues dans le logiciel Excel pour élaborer des tableaux et des diagrammes visualisant les résultats de notre étude empirique.

\footnotetext{
${ }^{4}$ Néanmoins, dans la suite de la recherche et vu le nombre relativement restreint de copies soumises à l'analyse, nous ne nous sommes limités qu'à l'analyse quantitative des articulateurs logiques.
}

XLinguae, Volume 14 Issue 4, October 2021, ISSN 1337-8384, eISSN 2453-711X 


\subsection{La discussion des résultats}

Les diagrammes ci-dessous montrent les résultats de notre analyse :

Figure 1 Les résultats de l'analyse : connecteurs exprimant le but

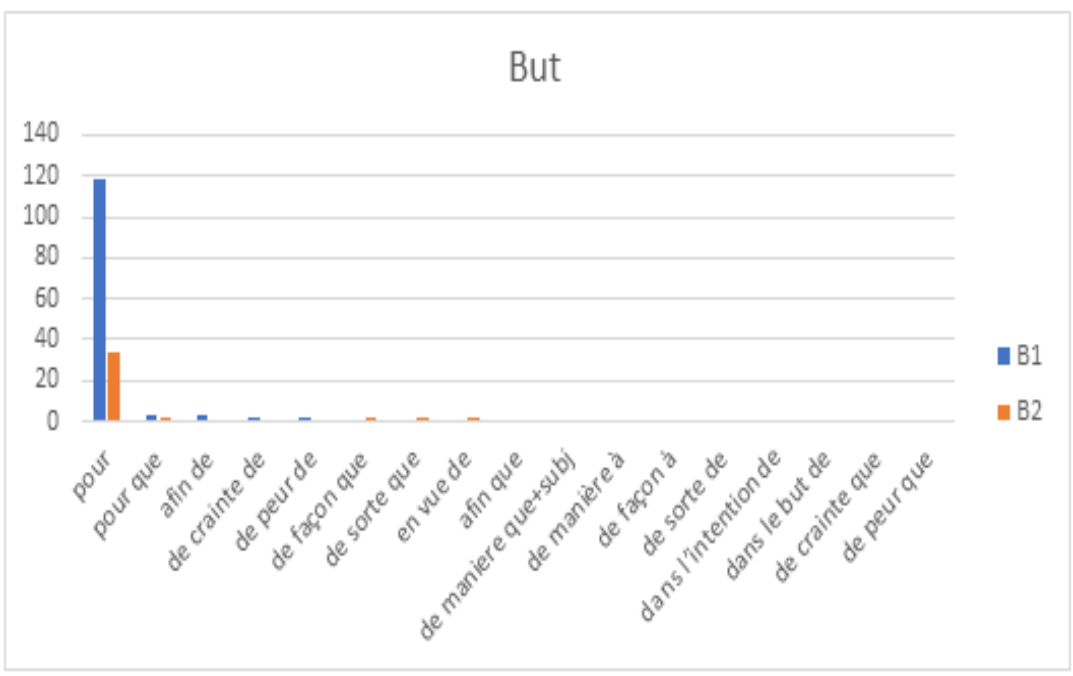

Figure 2 Les résultats de l'analyse : connecteurs exprimant la cause

\section{Cause}

- B1 $\mathrm{B} 2$

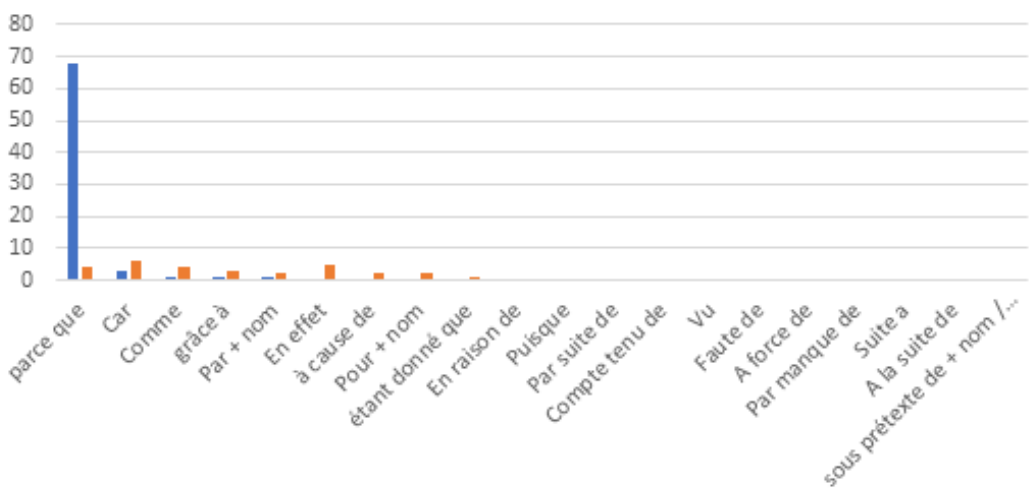


Figure 3 Les résultats de l'analyse : connecteurs exprimant la conséquence

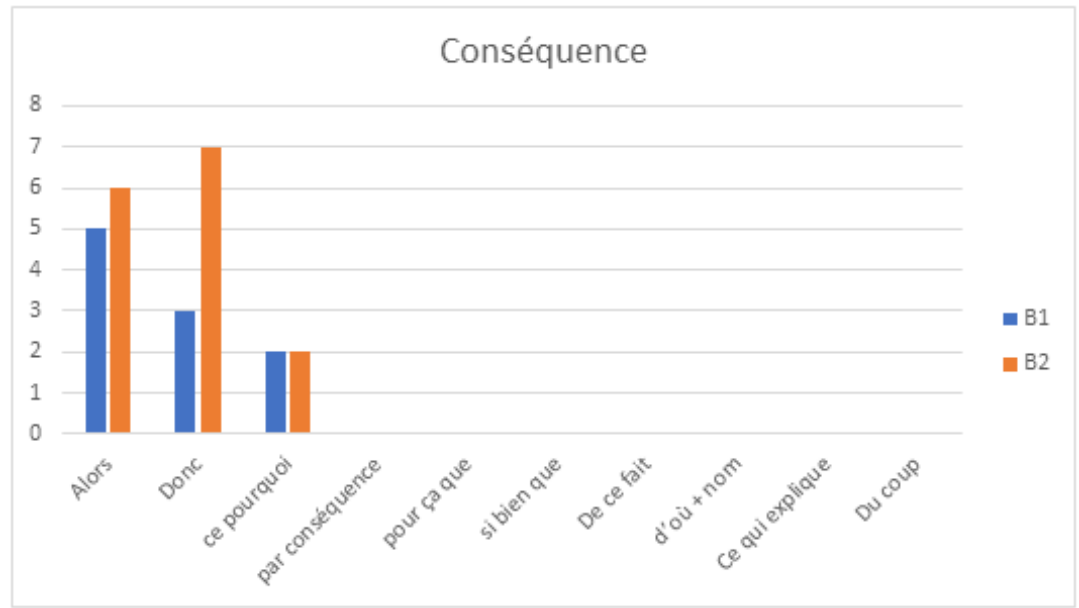

Figure 4 Les résultats de l'analyse : connecteurs exprimant l'opposition

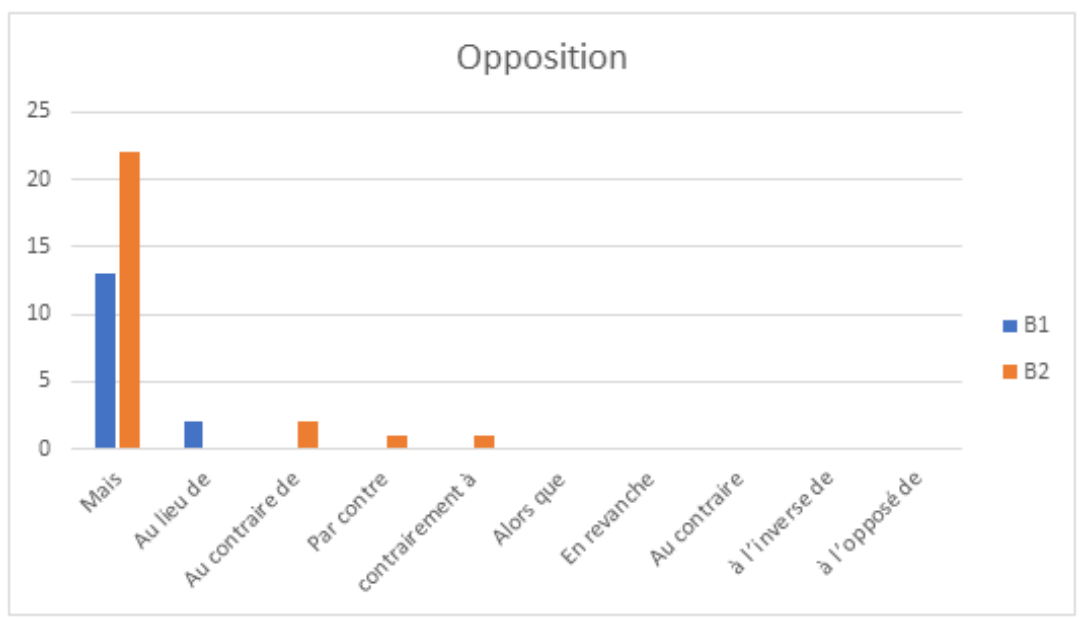

XLinguae, Volume 14 Issue 4, October 2021, ISSN 1337-8384, eISSN 2453-711X 


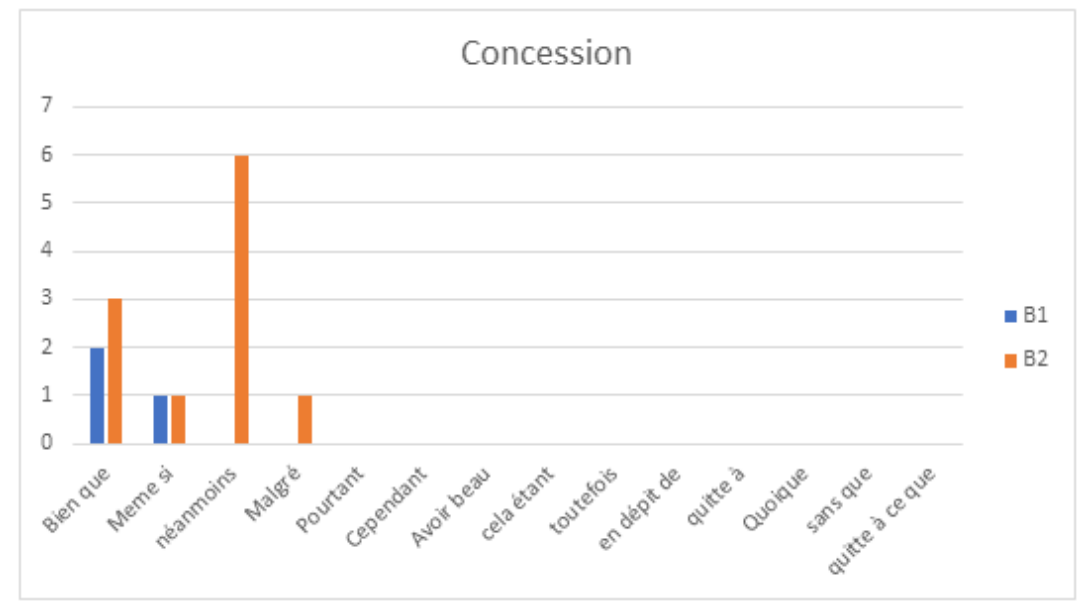

L'analyser des deux sous-corpus montrent les tendances suivantes :

- Les articulateurs logiques auxquels recourent les étudiants se présentant aux épreuves sont peu variés. En effet, pour enchaîner les différents éléments dans leurs textes, les candidats n'utilisent que 2-3 mots de liaison de chacune catégorie. Une grande gamme d'articulateurs n'est pas utilisé par les étudiants, ce qui est quand-même regrettable surtout au niveau B2 où la complexité langagière serait valorisée.

- Pour exprimer les relations logiques données, presque tous les étudiants (aussi bien ceux qui se sont présenté à l'épreuve B1 que ceux qui ont décidé de passer le test de français au niveau B2) ont recouru à des articulateurs prévus pour le niveau B1. Les articulateurs prévus pour le niveau B2 n'ont été utilisés que quelques fois. Cela peut s'expliquer probablement par le fait que les étudiants plus avancés n'ont pas voulu prendre de risque en situation d'examen, notamment avec un examen à enjeu élevé qui conditionnait leur réussite en $1^{\text {er }}$ cycle d'études universitaires.

- Seul le connecteur "néanmoins » a été utilisé 6 fois dans les copies au niveau B2.

- Un certain équilibre dans l'usage des articulateurs dans les copies au niveau B1 et B2 est à remarquer seulement dans les relations exprimant la conséquence.

- En général, les étudiants du niveau B2 possèdent une gamme plus large de connecteurs logiques que les étudiants représentant le niveau B1. Cependant, ces cas sont loin d'être nombreux.

- Les relations logiques exprimant le but, la cause et l'opposition sont dominées par les articulateurs les plus basiques, tels que " pour», "parce que » et « mais ».

\section{Les remarques conclusives}

Les hypothèses que nous avions avancées au départ de notre recherche n'ont pas été confirmées. En effet, les étudiants du niveau B2 n'utilisent pas de connecteurs logiques d'un niveau supérieur qui leur auraient permis d'exprimer leurs propos avec le degré de précision demandé à ce niveau et imposé par le type de tâche (activité de médiation de textes). Sans nul doute, ce résultat est dû à la situation d'examen 
pendant lequel les candidats préfèrent ne pas prendre de risque et rester dans l'ombre du niveau B1 (au moins pour les articulateurs) de peur de commettre des fautes qui pourraient affecter négativement leur réussite. Il nous semble donc pertinent de refaire cette recherche auprès d'un public plus large et pas forcément dans une situation de l'examen. Disposer d'un échantillon représentatif (niveau B1 et B2) nous permettrait de l'analyser non seulement dans une optique quantitative mais aussi de traiter les données recueillies selon les principes de l'approche qualitative. Ainsi, nous pourrions effectuer aussi une analyse comparative des connecteurs utilisés par les étudiants (corpus d'apprenants : niveau B1 et B2) par rapport aux connecteurs inclus dans un corpus didactique construit, par exemple, à la base des textes inclus dans les manuels de FLE (B1 et B2). Il serait également intéressant de comparer le corpus d'apprenants avec un corpus de référence, comme le corpus Frantexte et ses sous-corpus. Les démarches de recherche que nous venons de présenter à titre d'exemple nous permettraient de formuler des propositions de démarches didactiques qui seraient à mettre en œuvre en classe de FLE pour le public polonophone.

\section{Bibliographic references}

AKYUZ, A. et al. 2019. En contexte : Exercices de grammaire B1. Paris : Hachette FLE. ISBN 9782014016345

AKYUZ, A. et al. 2019. En contexte : Exercices de grammaire B2. Paris : Hachette FLE. ISBN 9782014016352

ASTON, G. 1997. Small and large corpora in language learning. In: J. Melia - B. Lewandowska-Tomaszczyk (Eds.), PALC '97 Proceedings. The First International Conference: Practical Applications in Language Corpora (pp. 51-62). Łódź: Łódź University Press. Available online: http://www.sslmit.unibo.it/ guy/wudj1.htm. ISBN: 978-0820447544

ATTABI, D. 2012. Apprentissage de l'écriture : problèmes liés à l'emploi des connecteurs. In : Synergies Algérie vol. 15, pp. 83-94. ISSN 2260-5029

BERNARDINI, S. 2000. Systematising serendipity: Proposals for concordancing large corpora with language learners. In: L. Burnard - T. McEnery (Eds.), Rethinking language pedagogy from a corpus perspective. Frankurt a. M. et al.: Peter Lang. pp. 225-234. ISBN 978-3631365540

CADRE EUROPEEN COMMUN DE REFERENCE POUR LES LANGUES. 2001. Strasbourg : Conseil de l'Europe / Paris : Les Éditions Didier. ISBN 227805075-3

CADRE EUROPEEN COMMUN DE REFERENCE POUR LES LANGUES. 2018. Volume complémentaire avec de nouveaux descripteurs. Strasbourg: Conseil de l'Europe.

CAQUINEAU-GUNDUZ, M-P. et al. 2005. Les 500 exercices de grammaire. Niveau B1. Paris : Hachette FLE. ISBN 9782011554338

CAQUINEAU-GUNDUZ, M-P. et al. 2007. Les 500 exercices de grammaire. Niveau B2. Paris : Hachette FLE. ISBN 9782011554383

CHAMBERS, A. 2005. Integrating corpus consultation procedures in language studies. In: Language Learning \& Technology, vol. 9., n. 2, pp. 111-125. ISSN 10943501

CHAMBERS, A. - O'SULlIVAN, I. 2004. Corpus consultation and advanced learners' writing skills in French. In: ReCALL, vol. 16, n. 1, pp. 158-172. ISSN 14740109

COBB, T. 1997. Is there any measurable learning from hands-on concordancing? In: System, vol. 25, pp. 301-315. ISSN 0346-251X

COBB, T. 1998. Breadth and depth of lexical acquisition with hands-on concordancing. In: Computer Assisted Language Learning, vol. 12, n. 4, pp. 345-360. ISSN 1744-3210

XLinguae, Volume 14 Issue 4, October 2021, ISSN 1337-8384, eISSN 2453-711X 
COBB, T. - GREAVES, C. - HORST, M. 2001. Can the rate of lexical acquisition from reading be increased? An experiment in reading French with a suite of on-line resources. A translated chapter in : P. Raymond - C. Cornaire (Eds.), Regards sur la didactique des langues secondes. Éditions logique. pp. 133-153. ISBN 9782893816753

CUQ, J.-P. 2002. Dictionnaire de la didactique du français langue étrangère et secondaire. Paris : Clé international. ISBN 978-2-09-033972-7

CUQ, J.-P. - GRUCA, I. 2012. Cours de didactique du français langue étrangère et seconde. Grenoble : Presses universitaire de Grenoble. ISBN 978-2706126772

DE SCHRYVER, G. M. 2002. Web for/as corpus: A perspective for the African languages. In: Nordic Journal of African Studies, vol. 11, n. 2, pp. 266-282. ISSN 1459-9465

ELALOUF, M.-L. - TREVISE, A. 2011. Le traitement des connecteurs dans les instructions officielles et les manuels (français L1 / anglais L2). In : Revue française de la linguistique appliquée, vol. 16, n. 2, pp. 121-140. ISSN 1875-368X

GASKELL, D. - COBB, T. 2004. Can learners use concordance feedback for writing errors? In: System, vol. 32, n. 3, pp, 301-319. ISSN 0346-251X

GOULLIER, F. 2019. Les clés du Cadre. Enjeux et actualités pour l'enseignement des langues aujourd'hui. Paris : Didier. ISBN 978-2-278-09525-4

HADLEY, G. 2002. Sensing the winds of change: An introduction to Data-Driven Learning. In: RELC Journal, vol. 33, n. 2, pp. 99-124. ISSN 1745-526X

HORST, M. - COBB, T. - NICOLAE, I. 2005. Expanding academic vocabulary with a collaborative on-line database. In: Language Learning \& Technology, vol. 9, n. 2, pp. 90-110. ISSN 1094-3501

KENNEDY, C. - MICELI, T. 2001. An evaluation of intermediate students' approaches to corpus investigation. In: Language Learning \& Technology, vol. 5, n. 3, pp. 77-90. ISSN 1094-3501

LEE, D. - SWALES, J. 2006. A corpus-based EAP course for NNS doctoral students: Moving from available specialized corpora to self-compiled corpora. In: English for Specific Purposes, vol. 25, pp. 56-75. ISSN 0889-4906

LEE, H. - WARSCHAUER, M. - LEE, J. H. 2017. The effects of concordance-based electronic glosses on L2 vocabulary learning. In: Language Learning \& Technology, vol. 21, n. 2, pp. 32-51. ISSN 1094-3501

MARINOV, S. 2013. Training ESP students in corpus use - challenges of using corpus-based exercises with students of non-philological studies. In: Teaching English with Technology, vol. 13, n. 4, pp. 49-76. ISSN 1642-1027

ROBERT, J.-P. - ROSEN, É. 2010. Dictionnaire pratique du CECR. Paris : Éditions OPHRYS. ISBN 978-2708012592

SALLES, M. 2006. Cohésion-cohérence : accords et désaccords. In : Corela 2006, DOI : 10.4000 .1426 ISSN 1638-573X

ST. JOHN, E. 2001. A case for using a parallel corpus and concordancer for beginners of a foreign language. In: Language Learning \& Technology, vol. 5, n. 3, pp. 185-203. ISSN 1094-3501

YOON, H. - HIRVELA, A. 2004. ESL student attitudes towards corpus use in L2 writing. In: Journal of Second Language Writing, vol. 13, pp. 257-283. ISSN 18731422

ZAJAC, J. 2004. Du communicatif au discursif. Apports de la pragmatique du discours à la didactique des langues étrangère. Exemple du FLE. Warszawa : Uniwersytet Warszawski - Instytut Romanistyki. ISBN 978-8388012808

Words: 6617

Characters: 43262 (24,03 standard pages) 
Radoslaw Kucharczyk

University of Warsaw

Krakowskie Przedmieście 26/28

00-927 Warszawa

Poland

Jaroslaw Krajka

Université Marie Curie-Skłodowska de Lublin

Pologne

Plac Marii Curie-Skłodowskiej 5

20-031 Lublin

Poland 